
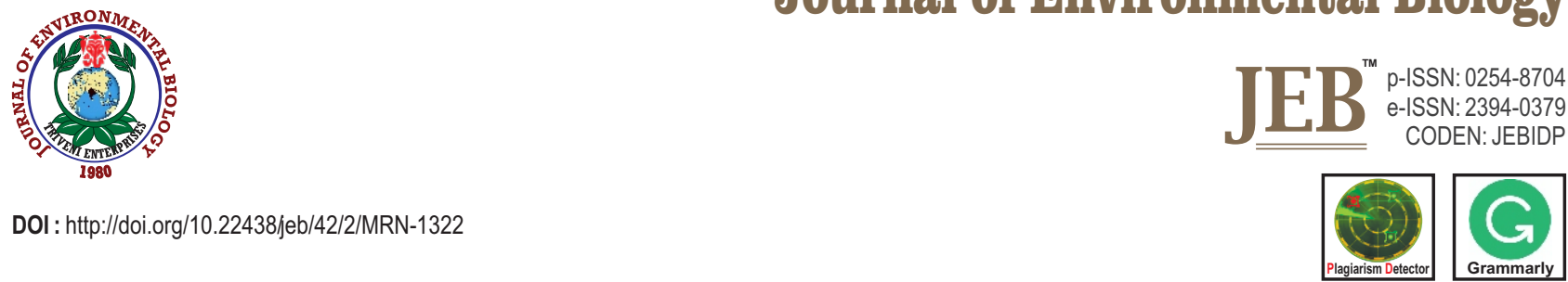

\title{
Development of species-specific marker and molecular diversity of Conogethes punctiferalis and Conogethes sahyadriensis (Lepidoptera: Crambidae)
}

\author{
V. Kammar', D. Sagar ${ }^{2 *}$, R.K. Chandel', P.R. Shashank ${ }^{2}$ and A.K. Chakravarthy ${ }^{3}$ \\ 'Department of Entomology, University of Agricultural Sciences, Gandhi Krishi Vigyana Kendra, Bangalore- 560065 , India \\ 2Division of Entomology, ICAR-Indian Agricultural Research Institute, New Delhi-110012, India \\ ${ }^{3}$ Division of Entomology and Nematology, ICAR-Indian Institute of Horticultural Research, Bangalore-560 089, India \\ *Corresponding Author Email : garuda344@gmail.com
}

\section{Abstract}

Aim: The aim of this study was to develop species specific marker for quick identification of two Conogethes species and to study their molecular diversity.

Methodology: Species-specific markers were developed using mitochondrial cytochrome oxidase 1 (COI) partial gene sequences of Conogethes punctiferalis and C. sahyadriensis and they were validated. Phylogenetic tree was constructed using MEGAX program, tree robustness was evaluated by bootstrapping with 1000 replicates with all sequences of $C$. punctiferalis and $C$. sahyadriensis.

Results: Bioinformatic analysis of $C$. punctiferalis and $C$. sahyadriensis $\mathrm{COI}$ sequences revealed that they have sufficient genetic variability to develop species specific marker. The COI based species-specific markers amplified an expected fragment size of 333 bp for $C$. punctiferalis and 522 bp for $C$. sahyadriensis, which clearly differentiate these two species.

Interpretation: The species specific marker developed in this study will be useful in quick identification of $C$. punctiferalis and $C$. sahyadriensis. The use of $\mathrm{COI}$ gene as a marker can provide a better estimate of genetic diversity, as it is maternally inherited and can be helpful to understand evolutionary processes.

Key words: Conogethes punctiferalis, C. sahyadriensis, MtCOI, Molecular diversity, Species-specific marker

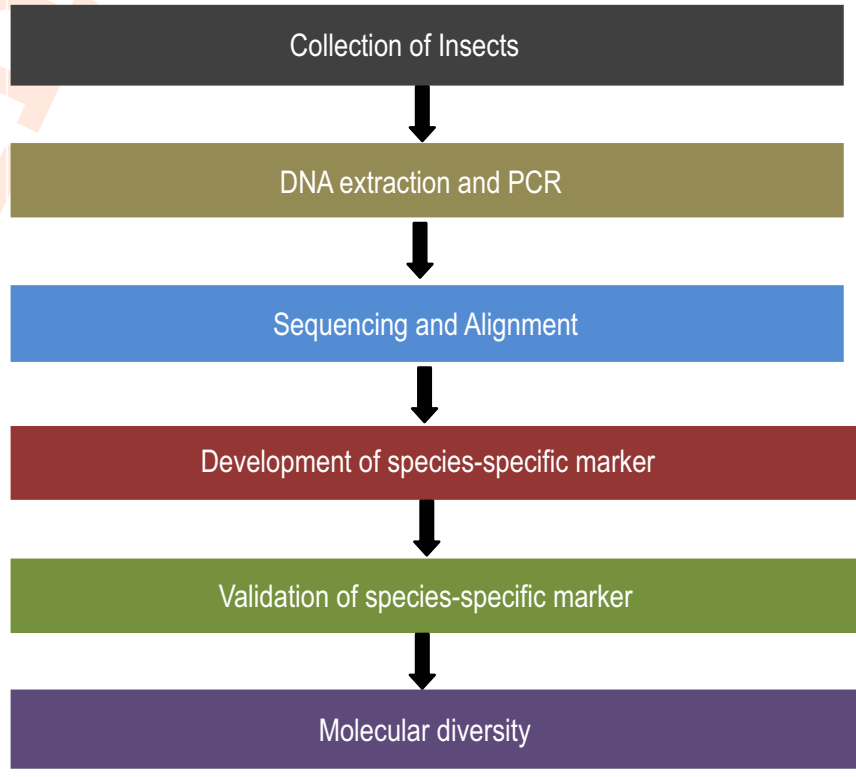

How to cite : Kammar, V., D. Sagar, R.K. Chandel, P.R. Shashank and A.K. Chakravarthy: Development of species-specific marker and molecular diversity of Conogethes punctiferalis and Conogethes sahyadriensis (Lepidoptera: Crambidae). J. Environ. Biol., 42, 271-279 (2021). 


\section{Introduction}

The shoot and fruit borers of genus Conogethes are lepidopteran pests infesting developing shoots and fruits. The genus Conogethes Meyrick, (1884) (Lepidoptera: Crambidae) is a large, taxonomically complex taxon of crambids distributed throughout the Oriental, Australian and tropical Asian regions (Shaffer et al., 1996, Inoue and Yamanaka 2006).Conogethes punctiferalis (Guénee) commonly called as yellow peach moth is one such species. Larvae of this moth are considered polyphagous attacking more than 120 wild and cultivated plants (Sekiguchi 1974). Type locality of C. punctiferalis is India and the species complex of Conogethes is poorly understood. Hampson (1896) reported 20 species of Dichocrocis and for distinguishing the species of Conogethes he studied the characters of wing venation and arrangements of black spots on the wings which are now considered of little value.

In absence of precise identification of C. punctiferalis, Koizumi (1960) in Japan for the convenience identified two types of C. punctiferalis viz., the Fruit Tree Type and Conifer Type from angiosperms and Pinaceae gymnosperms, respectively. Most of the studies on the Conogethes moths are in progress in Japan, China and Australia, and the species have been barcoded (Armstrong, 2010). There is a dire need for similar work on the Indian species of Conogethes considering their economic importance. Similarly, C. punctiferalis larvae in India infesting castor (Ricinus communis L.) and cardamom (Elettaria cardamomum Maton) have been identified as two different lineages based on mass-rearing techniques (Chakravarthy et al., 1991). Subsequently, Shashank et al. (2014a,b) accurately indicated differences in morphology, morphometry and molecular identification of male and female genitalia, larvae and pupae and in behaviour of above two species. Effect of interbreeding on offsprings between the two types of C. punctiferalis was also determined. Achille Guenee first described C. punctiferalis in 1854 and Sir George Hampson in 1896 included all species under the genus Dichocrocis based on morphological features. Since then Conogethes populations feeding on castor and cardamom have been included under the species punctiferalis. But in 2018, a new species C. sahyadriensis feeding on cardamom was delineated from closely related species of Conogethes (Shashank etal., 2018).

Selected molecular markers viz., Cytochrome b, 16S rRNA, 18S rRNA, 28S rRNA, 5.8S rRNA, internal transcribed spacers, elongation factor-1a, mitochondrial cytochrome oxidase-I (mtCOI) (Von Dohlen and Moran, 2000, Ji et al., 2003, Raboudi et al., 2005) have been employed by researchers for species identification and molecular phylogeny studies in Conogethes spp. Mitochondrial genes, because of maternal inheritance and reliable inter-specific variation (Savolainen et al., 2005), have been widely employed in studying molecular insect systematics (Simon et al., 1994). This is a first attempt to develop species-specific markers of Conogethes spp. in India. In the current study, molecular diversity analyses were carried out deploying mitochondrial (mitochondrial cytochrome oxidase-1) gene. The objective of the present study was to develop speciesspecific markers for lepidopteran pest, C. punctiferalis and $C$. sahyadriensis based on $\mathrm{mtCO}$ for life-stage independent identification and determination of phylogenetic relationship.

\section{Materials and Methods}

Stock cultures of two species of Conogethes populations were maintained in the laboratory at $23 \pm 2^{\circ} \mathrm{C}, 70-85 \%$ relative humidity and $16 \mathrm{~h}$ light and $8 \mathrm{hrs}$ dark period in Department of Entomology, UAS, GKVK, Benagaluru. Morphological identification of $C$. punctiferalis and $C$. sahyadriensis were carried out according to Shashank et al. (2018). For developing species- specific markers, the $C$. punctiferalis and $C$. sahyadriensis larvae were collected from castor (R. communis) and cardamom ( $E$. cardamomum), in Karnataka, South India. For molecular diversity studies, C. punctiferalis and C. sahyadriensis larvae were collected on select host plants from different locations in India (Table 1,2) and samples were preserved in ethyl alcohol and stored at $-20^{\circ} \mathrm{C}$.

Total DNA was isolated by DNeasy blood and tissue kit (QIAGEN GmbH, Germany) from individuals of Conogethes collected from diversified locations in India. The original specimens were preserved as voucher specimens in the Entomology Division, ICAR-Indian Agricultural Research Institute, Pusa campus, New Delhi. For development of speciesspecific marker and molecular diversity analyses, mitochondrial cytochrome oxidase-I (COI) gene was used. The genomic DNA was used as template in polymerase chain reaction (PCR) to amplify COI gene region with primers LCO1490 (F) 5' GGT CAA CAAATC ATAAAG ATA TTG G-3' and HCO 2198 (R) 5' TAAACT TCA GGG TGA CCA AAA AAT CA-3' (Folmer et al., 1994). Polymerase chain reaction was carried out in a thermal cycler (C1000, Bio-Rad, USA ) reaction with the following cycling parameters; initial denaturation at $94{ }^{\circ} \mathrm{C}$ for $4 \mathrm{~min}, 35$ amplification cycles $\left[94^{\circ} \mathrm{C}\right.$ for $1 \mathrm{~min}, 48^{\circ} \mathrm{C}$ or optimal annealing temperature $\left(\mathrm{Tm}^{\circ} \mathrm{C}\right)$ for $1 \mathrm{~min}, 72^{\circ} \mathrm{C}$ for $1 \mathrm{~min}$ ], followed by a final extension step at $72{ }^{\circ} \mathrm{C}$ for $5 \mathrm{~min}$. PCR was performed in $25 \mu \mathrm{l}$ total reaction volume containing $12.5 \mu \mathrm{l}$ of Thermo Scientific Dream Taq PCR Master Mix (2X), $10 \mu \mathrm{M}$ of primer (1 $\mu$ l each), nuclease free water $(8.5 \mu \mathrm{l})$ and DNA template $(2 \mu \mathrm{l})$.

The amplified products were electrophorosed on $1.0 \%$ agarose gel, stained with ethidium bromide with 100 bp DNA ladder (Invitrogen, Thermo Fisher Scientific, USA) and visualized in a UV gel documentation unit (Alpha Imager HP, USA). For the species-specific primers, as mentioned in Table 3, PCR mix and PCR cycling parameters were the same, except for the annealing temperature viz., $40^{\circ} \mathrm{C}$ for $60 \mathrm{sec}$. The PCR amplified single band was purified using a QIAquick PCR purification kit (QIAGEN $\mathrm{GmbH}$, Germany). Purified PCR products were sequenced in both forward and reverse directions with an automated sequencer (ABI Prism ${ }^{\circledR} 3730$ XL DNA Analyzer; Applied Biosystems, Maryland, USA). After obtaining the sequences, they were aligned using sequence alignment editor BioEdit version 7.0.5.3 (Hall 1999) and the homology search was carried out using BLAST (http://www. ncbi.nlm. nih. gov) to confirm the species identity. Sequences of Conogethes populations were deposited with the National Center for Biotechnology Information (NCBI) database and the accessions numbers were obtained (Table 1, 2). In order to develop the speciesspecific primer for C. punctiferalis and C. sahyadriensis, the differences in $\mathrm{COI}$ sequences of both the species were determined using the sequence alignment editor BioEdit version 7.0.5.3 (Hall, 1999). Five sets of forward and reverse 
primers were synthesized based on the variable regions in the aligned sequences of the same. The primers thus designed were validated both on identified Conogethes populations collected on the select host plants from field. PCR amplified fragments resulting from species-specific markers for Conogethes populations were further sequenced and analyzed as above. To validate the species-specific primer specificity, cross amplification was tried through PCR for both the primer sets developed in the current study. All the sequences established were analysed corresponding to $\mathrm{COI}$, using BioEdit. 7.0 program using Clustal W. The aligned sequences were further analysed using MEGA version X (Kumar et al., 2018). Neighbour-Joining (NJ) trees were constructed with Kimura-2-parameter (K2P) distance model (Kimura 1980). All the corresponding sequences of mitochondrial markers for Conogethes were deposited in the NCBI-GenBank.

\section{Results and Discussion}

The PCR amplicon of the same size (Approx. 700 bp) was amplified for C. punctiferalis and C. sahyadriensis and the sequences showed that the total nucleotide length was $587 \mathrm{bp}$ for the two candidate species. The BLAST search showed that the sequences had the similarity for the respective species. Alignment of the COI sequences for C. punctiferalis (JX064420) and C. sahyadriensis (JX064423) in BioEdit v.7.0 revealed that among 587 in 31 nucleotide positions showed variations amounting to approximately $5 \%$ difference between $\mathrm{C}$. punctiferalis and C. sahyadriensis (Fig. 1). Species-specific markers require only PCR that is rapid, inexpensive and accessible. Of the 5 primer sets identified each for $C$. punctiferalis and C. sahyadriensis, 1 primer set, viz., $\mathrm{SD}(\mathrm{CP})-\mathrm{F}$ and $\mathrm{SD}(\mathrm{CP})$ -
R and SD (CS) - F and SD (CS) -R, successfully identified C. punctiferalis and C. sahyadriensis, respectively (Table 3, Fig. 2). These species-specific markers amplified an expected fragment size of $333 \mathrm{bp}$ for $C$. punctiferalis and $522 \mathrm{bp}$ for $C$. sahyadriensis. These same fragments were sequenced and results showed that species-specific markers exhibited the similarity for respective species. Similar amplicon sizes were obtained from test specimens of Conogethes populations derived from select host plants and geographical position in India.

Validation of the species-specific primers so developed were made deploying PCR using SD (CP) - F and SD (CP) -R and SD (CS) - $F$ and SD (CS) -R primers and genomic DNA isolated from two test species of Conogethes, morphologically identified and deposited at the Division of Entomology, Indian Agricultural Research Institute, Pusa, New Delhi. No PCR reaction produced amplification, including non-specific amplicons, where the PCR mix and PCR cycling conditions were same, including annealing temperature, viz., $40^{\circ} \mathrm{C}$ for $60 \mathrm{sec}$ for all the reactions undertaken. However, the applicability of these primers on other populations of $C$. punctiferalis and $C$. sahyadriensis within and outside India is based on the variations in the nucleotide sequences in forward (6-23 for both $C$. punctiferalis and $C$. sahyadriensis) and reverse primer binding regions 321-339 for $\mathrm{C}$. punctiferalis and $508-528$ for $C$. sahyadriensis. A comparison of forward primer binding regions for C. punctiferalis (JX064420.1) with other existing $\mathrm{COI}$ sequences (sequences generated in the current study) showed that there were variations at $5^{\text {th }}$ position. Similarly, in the reverse primer binding region, there were variations at $285^{\text {th }}$ position. One accession had $T$ instead of $C$ in another accession. A comparison of forward primer binding region for $C$. sahyadriensis with existing $\mathrm{COI}$ sequences showed that there were no variations in any of the nucleotide positions in either regions.

Table 1: Analyzed samples of $C$. punctiferalis with description of host plant, site of collection and NCBI-Genbank accession numbers

\begin{tabular}{llll}
\hline Species & Host plant & Site of collection & NCBI-GenBank accessions \\
\hline C. punctiferalis & Ricinus communis L. & Junagadh, Gujarat, India & $\mathrm{Jx064411}$ \\
C. punctiferalis & Ricinus communis L. & Junagadh, Gujarat, India & $\mathrm{Jx064412}$ \\
C. punctiferalis & Ricinus communis L. & Ludhiana, Punjab, India & $\mathrm{J} \times 064413$ \\
C. punctiferalis & Ricinus communis L. & Ludhiana, Punjab, India & $\mathrm{J} 064414$ \\
C. punctiferalis & Ricinus communis L. & Chhindwara, Madhya Pradesh, India & $\mathrm{J} \times 064415$ \\
C. punctiferalis & Ricinus communis L. & Chhindwara, Madhya Pradesh, India & $\mathrm{J} \times 064416$ \\
C. punctiferalis & Ricinus communis L. & Hyderabad, Andhra Pradesh, India & $\mathrm{Jx064418}$ \\
C. punctiferalis & Ricinus communis L. & Hassan, Karnataka, India & $\mathrm{Jx064419}$ \\
C. punctiferalis & Ricinus communis L. & Hassan, Karnataka, India & $\mathrm{Jx064420}$ \\
\hline
\end{tabular}

Table 2: Analyzed samples of $C$. sahyadriensis with description of host plant, site of collection and NCBI-Genbank accession numbers

\begin{tabular}{|c|c|c|c|}
\hline Species & Host plant & Site of collection & NCBI-GenBank accessions \\
\hline C. sahyadriensis sp. nov. & Elettaria cardamomum Maton & Mudigere, Karnataka, India & Jx064422 \\
\hline C. sahyadriensis sp. nov. & Elettaria cardamomum Maton & Mudigere, Karnataka, India & Jx064423 \\
\hline C. sahyadriensis sp. nov. & Elettaria cardamomum Maton & Mudigere, Karnataka, India & Jx064424 \\
\hline C. sahyadriensis sp. nov. & Elettaria cardamomum Maton & Bengaluru, Karnataka, India & Jx064425 \\
\hline C. sahyadriensis sp. nov. & Elettaria cardamomum Maton & Bengaluru, Karnataka, India & Jx064426 \\
\hline C. sahyadriensis sp. nov. & Elettaria cardamomum Maton & Idduki, Kerala, India & $\mathrm{Jx} 064427$ \\
\hline C. sahyadriensis sp. nov. & Elettaria cardamomum Maton & Idduki, Kerala, India & Jx064428 \\
\hline
\end{tabular}


Table 3: Species specific molecular markers developed for C. punctiferalis and C. sahyadriensis

\begin{tabular}{llll}
\hline Species & Primer & Binding region (bp) & Product size (bp) \\
\hline C. punctiferalis & SD (CP) -F - 5'-AGGAACTTCCTTAAGTTT-3' & $6-23$ & 333 \\
& SD (CP) -R - 5'TCCACCATGTGCAATATTA'3 & $321-339$ & \\
C. sahyadriensis & SD (CS) -F - 5'-GGGACTTCCTTAAGTTT-3' & $6-23$ & 522 \\
& SD (CS) -R - 5'TGCTAATACTGGTAAAGATAA'3 & $508-528$ & \\
\hline
\end{tabular}

Table 4: Maximum Composite Likelihood Estimate of the pattern of nucleotide substitution from 11 populations of $C$. punctiferalis and 8 populations of $C$. sahyadriensis

\begin{tabular}{|c|c|c|c|c|c|c|c|}
\hline & A & & $T$ & & C & & G \\
\hline C. punctiferalis & C. sahyadriensis & C. punctiferalis & C. sahyadriensis & C. punctiferalis & C. sahyadriensis & C. punctiferalis & C. sahyadriensis \\
\hline$A-$ & - & 1.05 & 0.09 & 0.45 & 0.04 & 4.31 & 32.29 \\
\hline T 0.8 & 0.07 & - & - & 24.5 & 0.04 & 0.4 & 0.03 \\
\hline C 0.8 & 0.07 & 57.28 & 0.09 & - & - & 0.4 & 0.03 \\
\hline G 8.57 & 67.13 & 1.05 & 0.09 & 0.45 & 0.04 & - & - \\
\hline
\end{tabular}

Each entry shows the probability of substitution from one base (row) to another base (column) instantaneously. Only entries within a row should be compared. Rates of different transitional substitutions are shown in bold and those of transversional substitutions are shown in italics

Molecular identification using a species-specific marker is an advantage where there is cryptic species complex in the target species population. In Conogethes, there is a suspicion that within C. punctiferalis complex, the population feed on a wide range of host plants leading to host-mediated speciation across diversified habitats. In such species complex (there may be more than one species) in lepidoptera, the development of speciesspecific markers for $C$. punctiferalis and $C$. sahyadriensis are of immense significance. The species-specific markers enable even a non- specialist or non-taxonomist to identify the target species, i.e., C. punctiferalis and C. sahyadriensis at any developmental stage without sequencing and elaborate examination and time.

The maximum composite likelihood defines the sum of related log-likelihoods among the species components (Tamura et al., 2004). The biological data sets for COI were utilised for maximum composite likelihood estimate pattern of nucleotide substitution for $C$. punctiferalis sequences using MEGA.X (Kumar et al., 2018). The reliability of clustering pattern in the phylogenetic trees was determined by bootstrap test, with 1000 replications. Rates of different transition substitutions are shown in bold and those of transversion substitutions are shown in italics. The C. punctiferalis nucleotide frequencies were $0.343(\mathrm{~A}), 0.359$ $(\mathrm{T}), 0.164(\mathrm{C})$ and $0.133(\mathrm{G})$. The base composition of these gene fragments was biased toward Adenine $(A)$ and Thyamine $(T)$, which together constituted $70.2 \%$ of the total nucleotides. The overall transition (ti)/ transversion (tv) bias of $\mathrm{C}$. punctiferalis nucleotide sequence was $R=1.007$, where $R=\left[A^{*} G^{*} k 1+\right.$ $\left.T^{*} C^{*} k 2\right] /\left[(A+G)^{*}(T+C)\right]$. Codon positions included were $1^{\text {st }}+2^{\text {nd }}+$ $3^{\text {rd }}+$ Noncoding. For $C$. sahyadriensis, the nucleotide frequencies were 0.344 (A), 0.361 (T), 0.155 (C) and 0.14 (G) (Table 4).

The base composition of $\mathrm{COI}$ gene sequences was biased towards Adenine. Current analysis conducted using the sequences generated for both $C$. punctiferalis and $C$. sahyadriensis, revealed sequence variations. This suggests that genetic structuring in terms of species, host-associated or habitat-related genetic differences are associated with both $C$. punctiferalis and $C$. sahyadriensis. This was evidenced using $\mathrm{COI}$ sequences of other Conogethes populations. As discovered, genital variations of both sexes between $C$. punctiferalis and $C$. sahyadriensis are reflected in their genetic structural differences and can be the result of host-mediated speciation with associated environmental factors affecting the growth and development of Conogethes population.

The Neighbor-Joining tree was constructed using MEGA X (Kumar et al., 2018) with bootstrap support (1000), and Marwitzia dichocrocis (Hampson) was used as an out-group in the species analysis of Conogethes sp. $M$. dichocrocis is a closely related genus of Conogethes from Africa. In $C$. punctiferalis, $\mathrm{NJ}$ tree was constructed from nine generated sequences of $\mathrm{COI}$ gene across the host plants with one outgroup. Phylogeny results showed that $C$. punctiferalis sequences formed a separate clade showing high diversity within the species (Fig. 3A). It clearly indicated that C. punctiferalis populations are undergoing genetic pressure in response to its wide host range, may lead to host-mediated speciation within the $C$. punctiferalis complex in India and other country as well. In C. sahyadriensis, eight $\mathrm{CO}$ based sequences were generated from a group of select host plants and utilised for construction of NeighborJoining tree with one out-group. Phylogeny tree clearly revealed no genetic diversity within $C$. sahyadriensis and formed a single clade in the phylogeny (Fig. 3B). This clearly indicated that $C$. sahyadriensis is an oligophagous pest restricted to few zingiberaceous host plants without exhibiting any selection pressure due to host plants.

Rapid, accurate and timely identification of insects is important and challenging. Developing species-specific marker is 
C. punctiferalis (JX064420.1)

C. sahyadriensis (JX064423.1)

Clustal Consensus

C. punctiferalis (JX064420.1)

C. sahyadriensis (JX064423.1)

Clustal Consensus

C. punctiferalis (JX064420.1)

C. sahyadriensis (JX064423.1)

Clustal Consensus

C. punctiferalis (JX064420.1)

C. sahyadriensis (JX064423.1)

Clustal Consensus

C. punctiferalis (JX064420.1)

C. sahyadriensis (JX064423.1)

Clustal Consensus

C. punctiferalis (JX064420.1)

C. sahyadriensis (JX064423.1)

Clustal Consensus

C. punctiferalis (JX064420.1)

C. sahyadriensis (JX064423.1)

Clustal Consensus

C. punctiferalis (JX064420.1)

C. sahyadriensis (JX064423.1)

Clustal Consensus

C. punctiferalis (JX064420.1)

C. sahyadriensis (JX064423.1)

Clustal Consensus
10

20

30

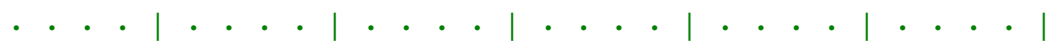
A T A G TA G G A A C C T T C C C T TA A G T T T A T T A A T T 30 A T A G T G G G A A C T T C C T T A A G T T T A T T A A T T 30 $* * * * * * * * * * * * * * * * * * * * * * * * * * * * * 29$

$40 \quad 50 \quad 60$

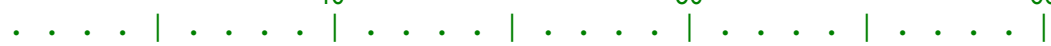
C G A G C T G A A T TA G G TA A T C C A G G A T C A T TA 60 C G A G C T G A A T TA G G TA A T C C A G G A T C A T TA 60 $* * * * * * * * * * * * * * * * * * * * * * * * * * * * * * 59$

$70 \quad 80 \quad 90$

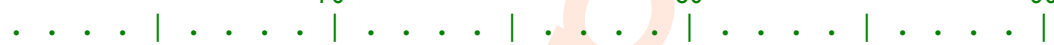
A T T G G A G A T G T C A A A T T T A T A A T A C A A T T $A T T G G A G A T G A T C A A A T T T A T A A T A C A A T T$ $* * * * * * * * * * * * * * * * * * * * * * * * * * * * * *$ 90

$100 \quad 110$ . . . G TA A C A G C T C A T G C T T T T A T T A T A A T T T T T $G T A A C A G C T C A T G C T T T T A T T A T A A T T T T C$ $* * * * * * * * * * * * * * * * * * * * * * * * * * * * * *$

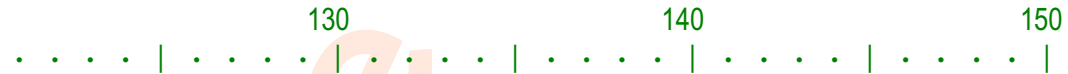

T T TA TA G TA A T A C C TA T T A T A A T T G G A G G T $T T T A T A G T A A T A C C T A T T A T A A T T G G A G G T$ $* * * * * * * * * * * * * * * * * * * * * * * * * * * * * * * * * *$

150

150 148

160 170

180

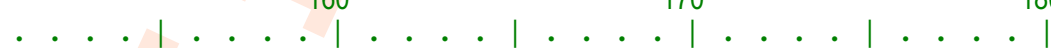
$T T T G G A A A T T G A T T A G T T C C T C T A A T A T T A$

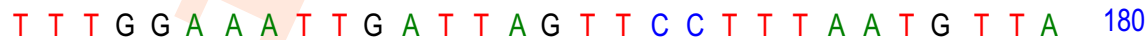
$* * * * * * * * * * * * * * * * * * * * * * * * * * * * * * 176$

$190200 \quad 210$

... . . . . . . . . . . . . . . . . . . . . . .

G G G G C C C C A G A T A T A G C T T T C C C T C G A A T A 210 G G A G C T C C A G A T A T A G C C T T T C C C C G A A TA 210 $* * * * * * * * * * * * * * * * * * * * * * * * * * 201$

$220 \quad 230 \quad 240$

A A T A A T A T A A G A T T T T G A T T A C T T C C C C C T 240 A A T A A T A T A A G A T T T T G A C T A C T T C C C C C $* * * * * * * * * * * * * * * * * * \quad * * * * * * * * * * * 230$

250

260

270

... . . . . . . . . . . . . . . . . . . . . . . . . . . .

T C A C T A A C T C T T T T A A T T T C C A G A A G A A T T 270

T C A T T A A C T C T T T TA A T T T C T A G A A G A A T T 270

$* * * * * * * * * * * * * * * * * * * * * * * * * * * * * 258$ 
C. punctiferalis (JX064420.1) C. sahyadriensis (JX064423.1) Clustal Consensus

C. punctiferalis (JX064420.1) C. sahyadriensis (JX064423.1) Clustal Consensus

C. punctiferalis (JX064420.1) C. sahyadriensis (JX064423.1) Clustal Consensus

C. punctiferalis (JX064420.1) C. sahyadriensis (JX064423.1) Clustal Consensus

C. punctiferalis (JX064420.1) C. sahyadriensis (JX064423.1) Clustal Consensus

C. punctiferalis (JX064420.1) C. sahyadriensis (JX064423.1) Clustal Consensus

C. punctiferalis (JX064420.1) C. sahyadriensis (JX064423.1) Clustal Consensus

C. punctiferalis (JX064420.1) C. sahyadriensis (JX064423.1) Clustal Consensus

C. punctiferalis (JX064420.1) C. sahyadriensis (JX064423.1) Clustal Consensus

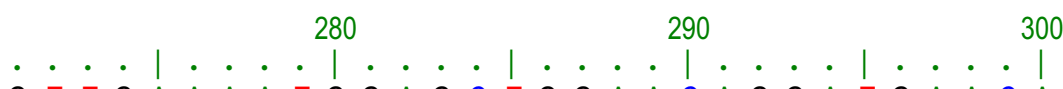

G T T G A A A A T G G A G C T G G A C A G G A T G A A C A 300 G T T G A A A A T G G A G A G G A A C A G G A T G A A C A 300 $* * * * * * * * * * * * * * * * * * * * * * * * * * * * * * 287$

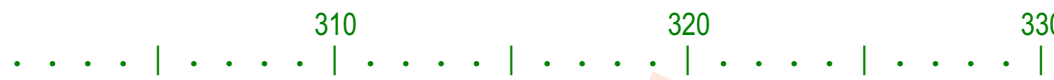

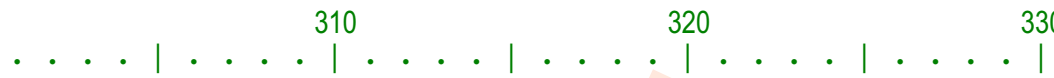

330

G TA T A C C C C C C T C T T T C A T C T A A T A T T G C A 330

G T A T A C C C C C C C C T T T C A T C C A A T A T T G C T 330

$* * * * * * * * * * * \quad * * * * * * * * \quad * * * * * * * * 314$

340

350

360

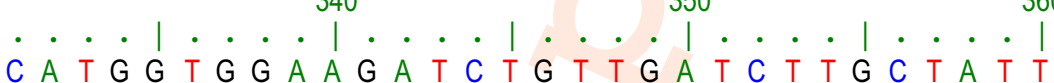

C A T A G T G G TA G A T C T G T T G A T C T C G C T A T C 360

$* * * * * * * * * * * * * * * * * * * * * * * * * * * 340$

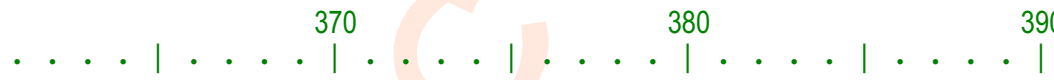

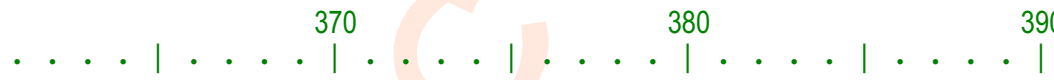

390

T T T T C C C T T C A T T T A G C G G G A A T T T C T T C T 390

T T T T C C C T T C A T C TA G C G G G A A T T T C C T C T 390

$* * * * * * * * * * * * * * * * * * * * * * * * * * 2 * * * 368$

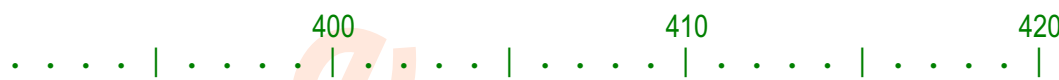

A T T T TA G A G C G A T TA A T T T C A T T A C A A C A 420

A T T T T A G G A G C A A T T A A T T T C A T C A C A A C A 420

$* * * * * * * * * * * *_{*} * * * * * * * * * * * * * * * * * 396$

... . . . . . . . . . . . . . . . . . . . . . . .

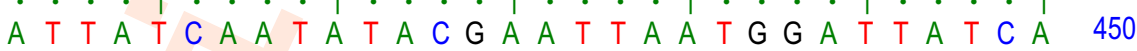

A T T A T T A A TA TA C G A A T TA A T G G A C T A T C A 450

$* * * * * * * * * * * * * * * * * * * * * * * * * * * * * * 424$

... | . . . . . . . . . . . . . . . . . . . . . . .

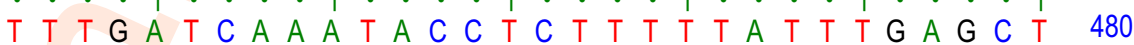

T T T G A T C A A A T A C C T C T T T T T A T T T G A G C C T 480

$* * * * * * * * * * * * * * * * * * * * * * * * * * * * * * 454$

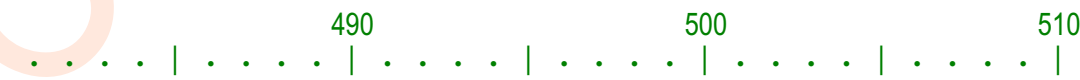

G TA G A A T TA C A G C T T TA T TA C T T C T T $\dot{T} \dot{T}$ A 510

G T A G G A A T T A C A G C T T T A T T A C T T C T T T T A 510

$* * * * * * * * * * * * * * * * * * * * * * * * * * * * * * 483$

$520530 \quad 540$

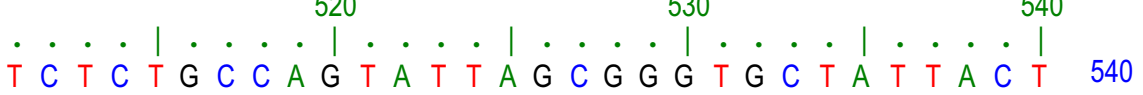

T C T T T A C C A G T A T T A G C A G G T G C T A T T A C T 540

$* * * * * * * * * * * * * * * * * * * * * * * * * * * * 510$ 
C. punctiferalis (JX064420.1)

C. sahyadriensis (JX064423.1)

Clustal Consensus

C. punctiferalis (JX064420.1)

C. sahyadriensis (JX064423.1)

Clustal Consensus
$550 \quad 560$

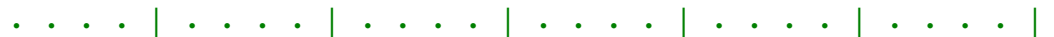

A T A C T T T TA A C A G A T C G TA A T C T TA A TA C T

A T A C C T T T TA A C A G A T C G T A A T C T T A A T A C T

570

570

540

Fig. 1: Consensus sequence of $587 \mathrm{bp}$ from the mitochondrial cytochrome oxidase I (COI) gene for $\mathrm{C}$. punctiferalis and C. sahyadriensis.

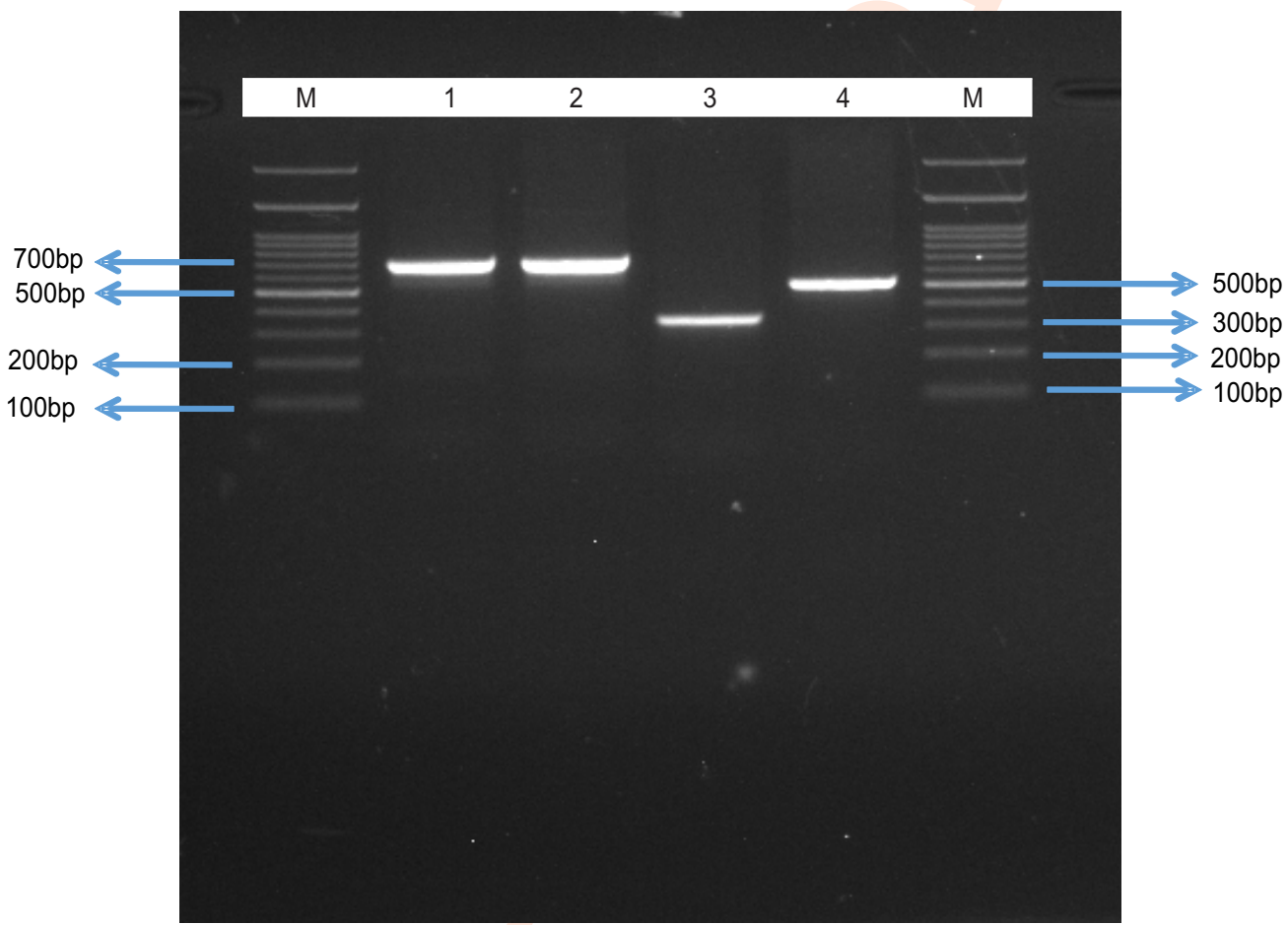

Fig. 2: Validation of species-specific markers for C. punctiferalis and C. sahyadriensis. M - 100bp DNA ladder (Invitrogen, Thermo Fisher Scientific) 1 and 2 - PCR amplified product (COI gene) of C. punctiferalis and C. sahyadriensis, respectively; 3: C. punctiferalis - specific molecular marker; 4: C. sahyadriensis-specific marker.

an easy, economical way of species discrimination in closely related species. This method is handy and independent of life stage, sex, polymorphism and other related factors. Rebijith et al. (2012) developed species-specific marker for identification of tea mosquito bug, Helopeltis species in India. The results showed that using species-specific marker Helopeltis antonii Signoret and Helopeltis thievora Waterhouse populations were differentiated. DNA barcoding and species-specific marker can also be used for identification of different species of cryptic mired bugs in India.

Reza and Khadijeh (2018) developed species-specific primer and single nucleotide polymorphism (SNP) and found a multiplex polymerase chain reaction as a molecular diagnostic tool to distinguish immature stages of most common and abundant species of cutworms viz., Agrotis ipsilon (Hufnagel), Agrotis exclamationis (Linnaeus) and Peridroma saucia (Hubner). There has been suspicion that at least two types of Adoxophyes honmai (Yasuda) exist in Korea. This moth attacks apple, peach, pear and tea. Moths have extreme morphological similarity and can be distinguished by developing new PCR primer sets.

Similarly species specific primers have also been developed and validated for different insect pests viz., two species of genus Scolytus (Amini and Hosseini, 2016), five stored 


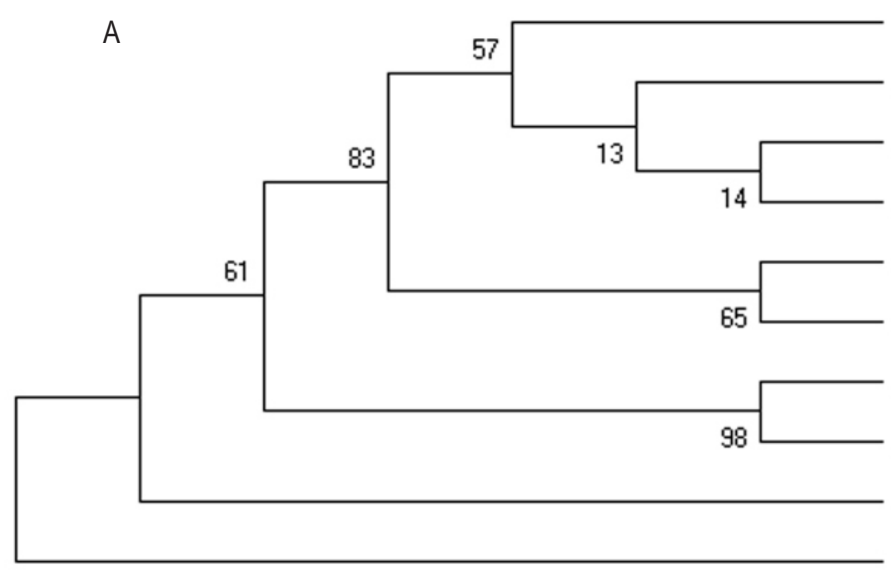

JX064420.1_Conogethes_punctiferalis

JX064416.1_Conogethes_punctiferalis

JX064415.1_Conogethes_punctiferalis

JX064414.1_Conogethes_punctiferalis

JX064418.1_Conogethes_punctiferalis

JX064411.1_Conogethes_punctiferalis

JX064419.1_Conogethes_punctiferalis

JX064413.1_Conogethes_punctiferalis

JX064412.1_Conogethes_punctiferalis

LT907834.1_Marwitzia_dichocrocis

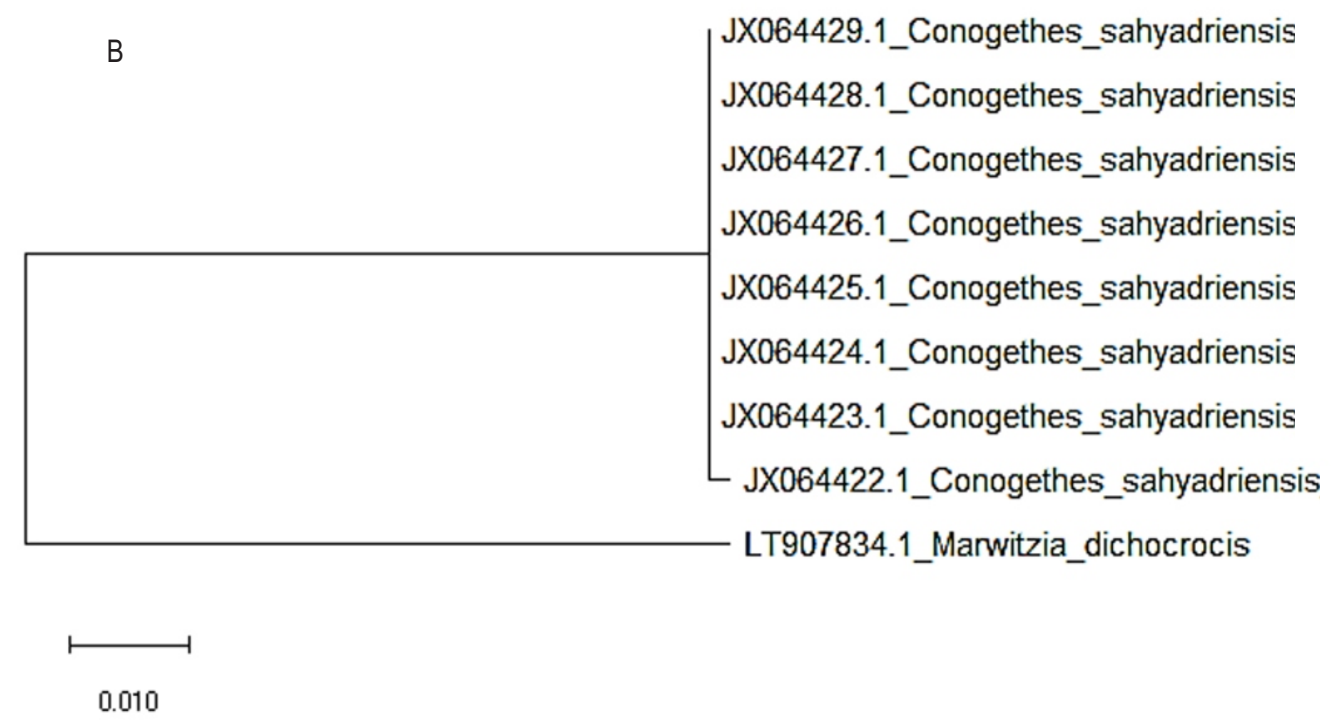

Fig. 3: Neighbour-joining phylogenetic tree of $C$. punctiferalis (A) and C. sahyadriensis (B) species based on the COI gene sequences. Marwitzia dichocrocis was used as an out group in the analysis.

product pests viz., Rhyzopertha dominica, Sitophilus granarius, S. oryzae, S. zeamais and Sitotroga cerealella (Sola et al., 2018) and four major thrips vectors viz., Thrips palmi, T. tabaci, Scirtothrips dorsalis, and Frankliniella schultzei (Jangra et al., 2020). These species specific primers are handy and quick tool in screening large number of samples and assessing the distribution profile of cryptic species.

This molecular identification technique with no enzyme digestion and sequencing step is convenient and rapid for identification of different species that are difficult to distinguish morphologically. The analysis of present study clearly showed that two species of Conogethes spp. remained skewed for a long time in India and also in neighbouring countries despite several studies on different aspects on two Conogethes populations. The importance of species-specific markers for species identification of $C$. punctiferalis and C. sahyadriensis amongst many other Conogethes species has been documented. From the present study, it can be concluded that developed markers helps in rapid, accurate, morphological and life-stage independent identification of C. punctiferalis and C. sahyadriensis which are morphologically indistinguishable. Undoubtedly, it helps in precise identification and in formulating effective management practices and also aids in developing appropriate quarantine protocols.

\section{Acknowledgments}

The senior author whole heartedly acknowledge the help received from Dr. S. Subramanian, Principal Scientist, Division of Entomology, ICAR-Indian Agricultural Research Institute, Pusa Campus, New Delhi, for providing laboratory facilities to conduct this work and I profoundly thank Mr. Prabhulinga T. Scientist, ICAR-Central Cotton Research Institute, Nagpur and Dr. Rani A.T., Scientist, ICAR-Indian Institute of Vegetable Research, Varanasi for their valuable guidance for preparing of manuscript. We are also thankful to the Department of Entomology, UAS, 
GKVK, Bengaluru as this work is a part of Ph. D thesis of first author.

\section{Add-on Information}

Authors' contribution: V. Kammar: Planning, execution of experiments; D. Sagar, P.R. Shashank: Planning, manuscript preparation; R.K. Chandel: Execution of experiments; A.K. Chakravarthy: Planning, final editing of manuscript.

Research content: The research content is original and has not been published elsewhere

Ethical approval: NotApplicable

Conflict of interest: The authors declare that there is no conflict of interest.

\section{Data from other sources: NotApplicable}

Consent to publish: All authors agree to publish the paper in Journal of Environmental Biology.

\section{References}

Amini, S. and R. Hosseini: A multiplex polymerase chain reaction based method for rapid identification of two species of the genus Scolytus Geoffroy (Col: Curculionidae: Scolytinae) in Iran. J. Entomol. Acarol. Res., 48, 11-15 (2016).

Armstrong, K. F.: DNA barcoding: A new module in New Zealand's plant biosecurity diagnostic toolbox. Bull. OEPP, 40, 91-100 (2010).

Chakravarthy, A.K., H. Honda and N.E. Thyagaraj: Comparison of containers for larval rearing in stalk and fruit feeding type of Conogethes punctiferalis (Guen.) (Lepidoptera: Pyralidae). Int. Sym. Plant. Crops, 9, 127-131(1991).

Folmer, O., M. Black, W. Hoeh, R. Lutz and R. Vrijenhoek: DNA primers for amplification of mitochondrial cytochrome $\mathrm{c}$ oxidase subunit I from diverse metazoan invertebrates. Mol. Marine Biol. Biotechnol., 3, 294-299 (1994).

Hall, T.A.: BioEdit: A user-friendly biological sequence alignment editor and analysis program for Windows 95/98/NT. Nucl. Acids. Symp. Ser., 41, 95-98 (1999).

Hampson, G.F.: The Fauna of British India including Ceylon and Burma (Moths). Taylor and Francis, London, pp. 594 (1896).

Inoue, $\mathrm{H}$. and $\mathrm{H}$. Yamanaka: Redescription of Conogethes punctiferalis (Guenée) and descriptions of two new closely allied species from Eastern Palaearctic and Oriental Regions (Pyralidae: Pyraustinae). Tinea, 19, 80-91 (2006).

Jangra, S., A. Mittal, H. Dhall, R. K. Jain and A. Ghosh: A multiplex PCR assay for rapid identification of major tospovirus vectors reported in India. BMC Genom., 21, 170 (2020).

Ji, Y.J., D.X. Zhang and L.J. He: Evolutionary conservation and versatility of a new set of primers for amplifying the ribosomal internal transcribed spacer regions in insects and other invertebrates. Mol. Ecol. Notes, 3, 581-585 (2003).
Kimura, M.: A simple method for estimating evolutionary rate of base substitutions through comparative studies of nucleotide sequences. J. Mol. Evol., 16, 111-120 (1980).

Koizumi, K.: Two forms of Dichocrocis punctiferalis (Guenee) presumably representing separate species. The main purport of a lecture the $20^{\text {th }}$ annual meeting of the Entomological Society of Japan., pp. 8-9 (1960).

Kumar, S., G. Stecher, M. Li, C. Knyaz and K. Tamura: MEGA X: Molecular evolutionary genetics analysis across computing platforms. Mole. Biol. Evolu., 35, 1547-1549 (2018).

Meyrick, E.: On the classification of the Australian Pyralidina. Trans. Ento. Soc. Lond., 1884, 277-350 (1884).

Raboudi, F., P. Chavigny, M. Marrakchi, H. Makni, M. Makni and M.F. Vanlerberghe: Characterization of polymorphic microsatellite loci in the aphid species Macrosiphum euphorbiae (Hemiptera: Aphididae). Molec. Ecol. Notes, 5, 490-492 (2005).

Rebijith, K.B., R. Asokan, N.K. Krishna Kumar, K.K. Srikumar, V.V. Ramamurthy and P. Shivarama Bhat: DNA barcoding and development of species-specific markers for the identification of tea mosquito bugs (Miridae: Heteroptera) in India. Environ. Entomol., 41, 1239-1245(2012).

Reza, H. and M. Khadijeh: A multiplex PCR method for identification of two common true cutworm species (Lepidoptera: Noctuidae) tested in the central plain of Guilan province, Iran, Zootaxa, 4420, 243-250 (2018).

Savolainen, V., R.S. Cowan, A. P. Vogler, G.K. Roderick and R. Lane: Towards writing the encyclopaedia of life: an introduction to DNA barcoding. Phil. Trans. Roy. Soc. London, B, Biol. Sci., 360, 18051811(2005).

Sekiguchi, K.: Morphology, biology and control of the yellow peach moth, Dichocrocis punctiferalis Guenée (Lepidoptera: Pyraustidae). Bull. Ibaraki Horticul. Expe. Station, Special Issue., 89, 1-90 (1974).

Shaffer, M., E.S. Nielsen and M. Horak: Pyraloidea. In: Checklist of the Lepidoptera of Australia. Monograph of Australia Lepidoptera, 4,164-199(1996).

Shashank, P.R., A.K. Chakravarthy, R. Chandrashekharaiah and K.R.M. Bhanu: Behavioural studies on shoot and fruit borer, Conogethes punctiferalis Guenée, (Crambidae: Lepidoptera) host-associated populations reveal occurrence of cryptic species. Entomol. Gener., 35, 103-115(2014a).

Shashank, P.R., A.K. Chakravarthy, B.R. Raju and K.R.M. Bhanu: DNA barcoding reveals the occurrence of cryptic species in hostassociated population of Conogethes punctiferalis (Lepidoptera: Crambidae). Appli. Entomol. Zool., 49, 283-295 (2014b).

Shashank, P. R., V. Kammar, R. Mally and A.K. Chakravarthy: A new Indian species of shoot and capsule borer of the genus Conogethes (Lepidoptera: Crambidae), feeding on cardamom. Zootaxa, 4374, 215-234 (2018).

Simon, C., F. Frati, A. Bechenbach and B. Crespi: Evolution, weighting, and phylogenetic utility of mitochondrial gene sequence and compilation of conserved polymerase chain reaction primers. Ann. Entomol. Soc. Am., 87, 651-701(1994).

Sola, M., J. Riudavets and N. Agusti: Detection and identification of five common internal grain insect pests by multiplex PCR. Food Control, 84, 246-54 (2018).

Von Dohlen, C.D. and N.A. Moran: Molecular data support a rapid radiation of aphids in the Cretaceous and multiple origins of host alternation. Biol. J. Linn. Soc., 71, 689-717 (2000). 